\title{
Pseudo-Univariate Calibration Based on NIR Spectroscopy in the Determination of Anthocyanins and Antioxidant Activity in Grape Juices
}

\author{
Karla K. Beltrame, ${ }^{a}$ Thays R. Gonçalves, ${ }^{a}$ Paulo H. Março, ${ }^{b}$ Sandra T. M. Gomes, ${ }^{a}$ \\ Makoto Matsushita ${ }^{a}$ and Patrícia Valderrama ${ }^{\circledR *, b}$ \\ ${ }^{a}$ Universidade Estadual de Maringá (UEM), 87020-900 Maringá-PR, Brazil \\ ${ }^{b}$ Universidade Tecnológica Federal do Paraná(UTFPR), 87301-899 Campo Mourão-PR, Brazil
}

\begin{abstract}
This work shows an alternative methodology based on a portable near-infrared (NIR) spectroscopy coupled to independent components analysis (ICA) in a pseudo-univariate calibration way to determine total anthocyanins (TA) concentration and antioxidant activity (AA) in whole grape juice. To this, the scores proportions more related to TA and AA were plotted against TA and AA obtained by its respective references methodology to build pseudo-univariate calibration models with correlation coefficients of 0.9699 and 0.9814 , respectively. From the results, it is possible the suggestion that NIR spectra coupled to ICA enable to overcome interferences using first-order data and work properly when there is enough selectivity for the analyte profile in the sample data.
\end{abstract}

Keywords: first-order data, DPPH, anthocyanins, curve resolution, spectroscopy

\section{Introduction}

Phenolic compounds have received prominence in the scientific and popular community due to its antioxidant activity, ${ }^{1}$ anticancer, antimicrobial, and anti-inflammatory properties. ${ }^{2}$ Also, it can prevent diabetes and obesity by inhibiting some enzymes. ${ }^{3}$

Grape juices are abundant in polyphenols, ${ }^{4}$ such as anthocyanins, which are present mainly as cyanidin, peonidin, petunidin, and malvidin, being widely known and investigated for their bioactive properties. ${ }^{5}$

Due to its benefits to human health, research related to antioxidant activities and flavonoids have increased significantly in the last decades, ${ }^{6-8}$ since the population has given more attention to food composition and quality. However, current reference methods for the determination of total anthocyanins and antioxidant activity require sample preparation, handling of toxic chemical reagents, generating chemical waste, as well as being sample destructive and expensive. ${ }^{9,10}$ Furthermore, considering that miniaturization and simplification of analytical procedures are trends in analytical chemistry, ${ }^{11}$ near-infrared (NIR) spectroscopy combined with chemometric tools have been widely used in food analysis, performing simple,

*e-mail: pativalderrama@gmail.com, patriciav@utfpr.edu.br fast, low-cost, nondestructive, simultaneous analyses and characterization of multiple components. ${ }^{12}$

The NIR regions present characteristics such as many weak and overlapping peaks of overtone and combination bands, so its exact assignments are difficult. However, this analytical technique reaches ample development with the technological revolution, by coupling the NIR spectra with chemometrics. ${ }^{13}$ In this sense, by comparing NIR calibrations performed with curve resolution tools and conventional multivariate calibration (performed, for example, from partial least squares) several advantages can be highlighted, detaching the possibility of isolating the contribution of the analyte even in in the presence of uncalibrated interferents, ${ }^{14,15}$ an important feature that lets to deal with the complexity of our matrix by eliminating the need for including the interferences in the calibration step.

Multivariate calibration models based on the resolution tools are mathematically simpler and can be built from a reduced number of samples. This kind of application is named pseudo-univariate calibration models due to univariate similarity. However, the data used are originally multivariate. ${ }^{11,16}$

Considering the numerous advantages, this work aimed to use the chemometric tool of independent components 
analysis (ICA) for pseudo-univariate calibration in the determination of total anthocyanins (TA) and antioxidant activity (AA) in whole grape juice from NIR spectra.

\section{Experimental}

\section{Samples}

Whole grape juices were acquired from different markets at Campo Mourão, Paraná, Brazil, to ensure that it has enough variability. Nine samples from different brands were analyzed.

\section{Total anthocyanins (TA)}

The TA was estimated according to the Association of Official Analytical Chemists (AOAC, method 2005.02). ${ }^{17}$ Different buffer systems ( $\mathrm{pH}$ 1.0, $0.025 \mathrm{~mol} \mathrm{~L}^{-1}$ potassium chloride (Vetec, Rio de Janeiro, Brazil), and pH 4.5, $0.4 \mathrm{~mol} \mathrm{~L}^{-1}$ sodium acetate (Vetec, Rio de Janeiro, Brazil)) were prepared, and $1 \mathrm{~mL}$ of each buffer was added in $1 \mathrm{~mL}$ of whole grape juice. The absorbance of the diluted samples, in both buffers, was measured at two different wavelengths 520 and $700 \mathrm{~nm},{ }^{17}$ with a portable UltravioletVisible (UV-Vis) spectrophotometer (Ocean Optics, Tampa, Florida, USA) and a quartz cuvette with $1 \mathrm{~mm}$ optical path. The TA concentration was expressed in cyanidin3-O-glucoside equivalent, ${ }^{18}$ according to the equation 1 :

$\mathrm{TA}\left(\mathrm{mg} \mathrm{L}^{-1}\right)=\frac{\mathrm{A} \times \mathrm{MW} \times \mathrm{DF} \times 10^{3}}{\varepsilon \times 1}$

where, $\mathrm{A}=\left(\mathrm{A}_{520}-\mathrm{A}_{700}\right)_{\mathrm{pH} 1.0}-\left(\mathrm{A}_{520}-\mathrm{A}_{700}\right)_{\mathrm{pH} 4.5}$; $\mathrm{MW}$ : molecular weight of cyanidin-3-O-glucoside; DF: dilution factor; $10^{3}$ : factor for conversion from $\mathrm{g}$ to $\mathrm{mg}$; $\varepsilon=26.900 \mathrm{~L} \mathrm{~mol}^{-1} \mathrm{~cm}^{-1}$ extinction coefficient for cyanidin3-O-glucoside; 1: path length (1 mm).

\section{Antioxidant activity (AA)}

The antioxidant activity of the whole grape juices was determined using the DPPH (2,2-diphenyl-1-picrylhydrazyl, Sigma-Aldrich, St. Louis, MO, USA) radical scavenging method. ${ }^{19}$ For this determination, a calibration curve was prepared using the ethanolic DPPH solution (0 to $\left.60 \mu \mathrm{mol} \mathrm{L}^{-1}\right)$. The absolute ethanol was acquired from Vetec (Rio de Janeiro, Brazil). In a dark environment, it was mixed $0.1 \mathrm{~mL}$ of grape juice with $3.9 \mathrm{~mL}$ of $60 \mu \mathrm{mol} \mathrm{L}^{-1} \mathrm{DPPH}$ solution. The absorbance for the mixtures was measured at $515 \mathrm{~nm}$, as suggested by the AOAC (method 2012.04). ${ }^{17}$ The results were expressed as DPPH equivalents $\left(\mu \mathrm{mol} \mathrm{L}{ }^{-1}\right)$.

\section{NIR spectroscopy}

The NIR spectra were recorded (in triplicate and the mean considered) in the range from 900 to $1700 \mathrm{~nm}$ (32 scans, $6 \mathrm{~nm}$ resolution), by using a portable MicroNIR (1700 spectrometer JDSU, San José, California, USA), and a glass cuvette. Data were handled by using MATLAB software version R2007b ${ }^{20}$ and the ICA resolution tool, whose mathematical steps are described in several articles, ${ }^{21,22}$ and not present here. Figure 1 shows a scheme of how the pseudo-univariate calibration curve for anthocyanins and antioxidants were obtained by using ICA from NIR spectroscopy.

\section{Results and Discussion}

The preprocessed NIR spectra of whole grape juice are presented in Figure 2. The preprocessing was done through the Savitsky-Golay algorithm, ${ }^{23}$ based on the first derivative (7 points and first-order polynomial).

From the literature, ${ }^{24}$ NIR spectra of samples rich in anthocyanins present a second overtone of the $\mathrm{O}-\mathrm{H}$ stretch at $982 \mathrm{~nm}$, a first overtone of the $\mathrm{O}-\mathrm{H}$ stretch at $1456 \mathrm{~nm}$, and combination bands of the asymmetric and scissor stretch O-H vibrations at $1940 \mathrm{~nm}$. Sugar-related absorption bands were characterized at 926 and $1208 \mathrm{~nm} .{ }^{24}$ On the other hand, NIR absorptions, related to antioxidant activity determined from the DPPH method, were verified at $950,1025,1125$ and $1310 \mathrm{~nm} .{ }^{11}$

Parallel to the NIR spectra recorder, the reference methods to determine TA and AA were applied to the same samples. The achieved results are shown, on the crescent concentration order, in Table 1.

For the preprocessed NIR spectra, the percentage of variance captured by principal component analysis (PCA) was employed to estimate the mathematical rank from the data set (Figure 3). The aim of PCA, in this case, is to capture variations and extract significant systematic trends in data sets for samples with large amounts of variables. In this way, PCA has been employed to suggest the rank when resolution methods are used. ${ }^{11,24}$ This occurs due to the bilinear data decomposition performed in PCA under the constraints of orthogonality (for vectors in both $\mathbf{T}$ and $\mathbf{P}^{\mathrm{T}}$ ), normalization of vectors in $\mathbf{P}^{\mathrm{T}}$, and in the directions of maximum explained variances for the successively extracted components. Under these constraints, obtained solutions are unique, but it lacks direct physical meaning. These characteristics make PCA useful for data interpretation but not for species resolution..$^{25}$

Based on the observations in the variance captured by each principal component (PC), the suggestion was rank equal 3, with $99.29 \%$ of cumulative explained variance. 
(a)

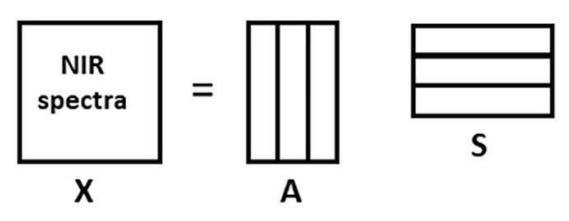

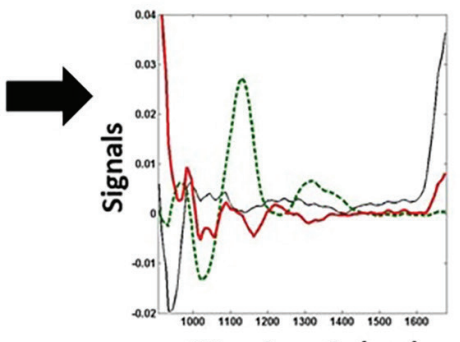

Wavelength $(\mathrm{nm})$ (b)

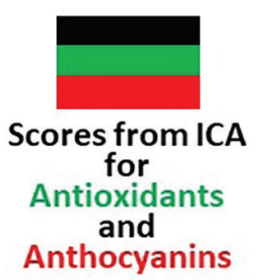

Anthocyanins

(c)
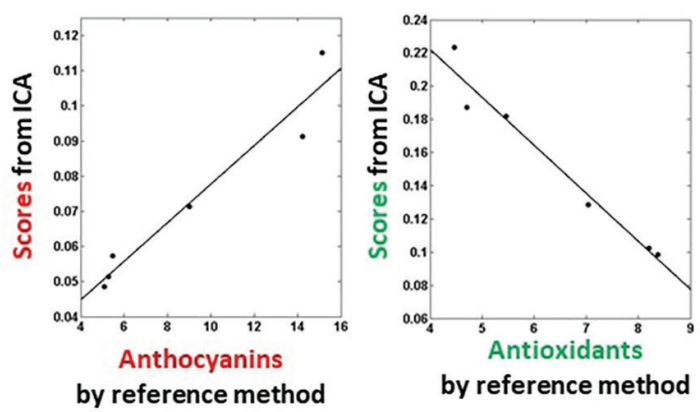

Figure 1. Scheme to perform a pseudo-univariate calibration curve for anthocyanins and antioxidants by using the NIR/ICA. (a) Deconvolution of the NIR spectra by ICA. (b) Representation of signals and scores for the constituents (anthocyanins, antioxidants, and interferent). (c) Pseudo-univariate calibration curve for anthocyanins and antioxidants determination.

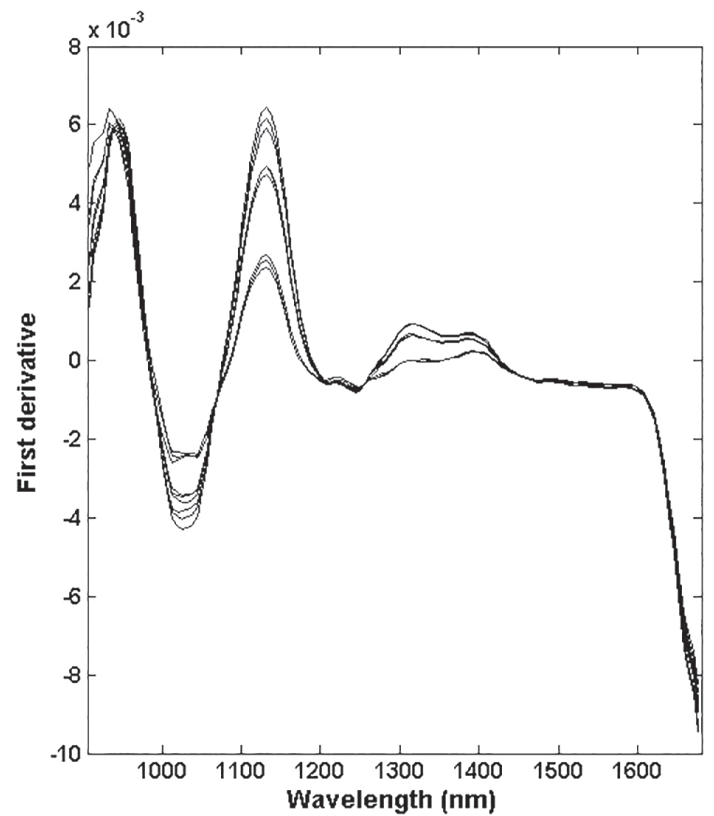

Figure 2. NIR spectra first derivative for grape juice samples.

ICA, with a rank equal to three, was applied to the NIR data set after the first derivative searching to signals resolution and scores proportions for total anthocyanins and antioxidant activity in the whole grape juice.

The ICA result is presented in Figure 4, which shows
Table 1. Results for the TA and AA by reference methods

\begin{tabular}{lc}
\hline $\begin{array}{l}\text { TA by reference methodology / } \\
\left(\mathrm{mg} \mathrm{L}^{-1}\right)\end{array}$ & $\begin{array}{c}\text { AA by reference methodology / } \\
(\mu \mathrm{M} \mathrm{DPPH})\end{array}$ \\
\hline 5.08 & 4.46 \\
5.27 & 4.70 \\
5.37 & 5.29 \\
5.46 & 5.46 \\
8.82 & 5.54 \\
9.01 & 7.04 \\
11.02 & 7.96 \\
14.23 & 8.21 \\
15.15 & 8.38 \\
\hline
\end{tabular}

TA: total anthocyanins; AA: antioxidant activity; DPPH: 2,2-diphenyl1-picrylhydrazyl.

the solved signal and scores proportions more related to total anthocyanins, and for the antioxidants that promote the reaction with DPPH to determine AA.

The pseudo-univariate calibration models to determine TA and AA in whole grape juices (Figure 5) were obtained from the scores proportions. To this, the scores proportions more related to anthocyanins for six samples were plotted against TA concentration determined by reference methodology. On the other hand, the scores proportions 


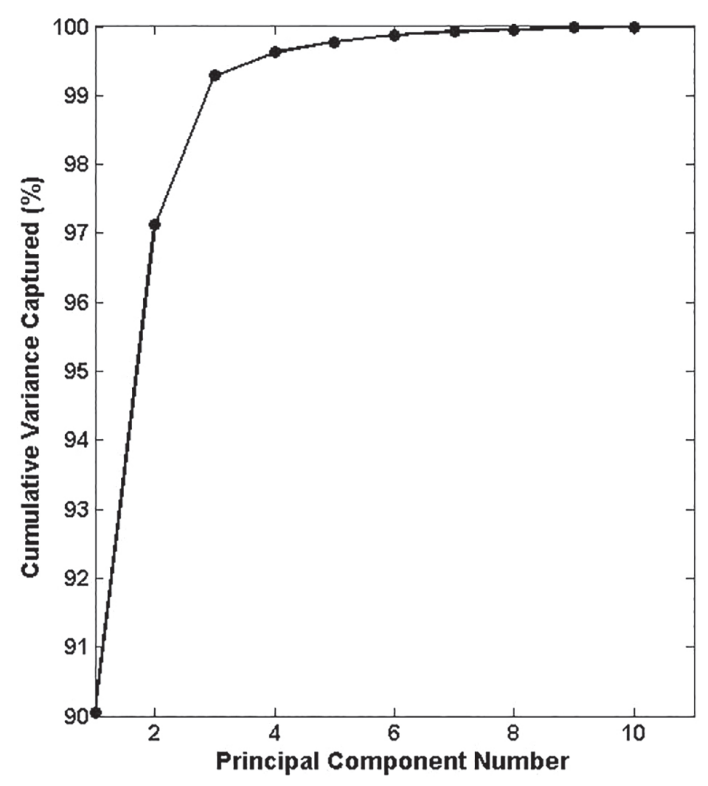

Figure 3. Cumulative variance captured by PCA.

more related to antioxidants were plotted against AA determined by DPPH methodology.

From the pseudo-univariate calibration curves, it is possible to observe a positive inclination for TA and a negative inclination for AA. In the DPPH analysis, the smaller the concentration needed to inhibit $50 \%$ of DPPH radical, the greater the antioxidant capacity of the sample. The pseudo-univariate models presented correlation coefficients of 0.9699 and 0.9814 for TA and AA determination, respectively. These models were employed to the determination of TA and AA in three independent samples, and the results are presented in Table 2.

Pseudo-univariate calibration models were previously employed successfully (after each application, under

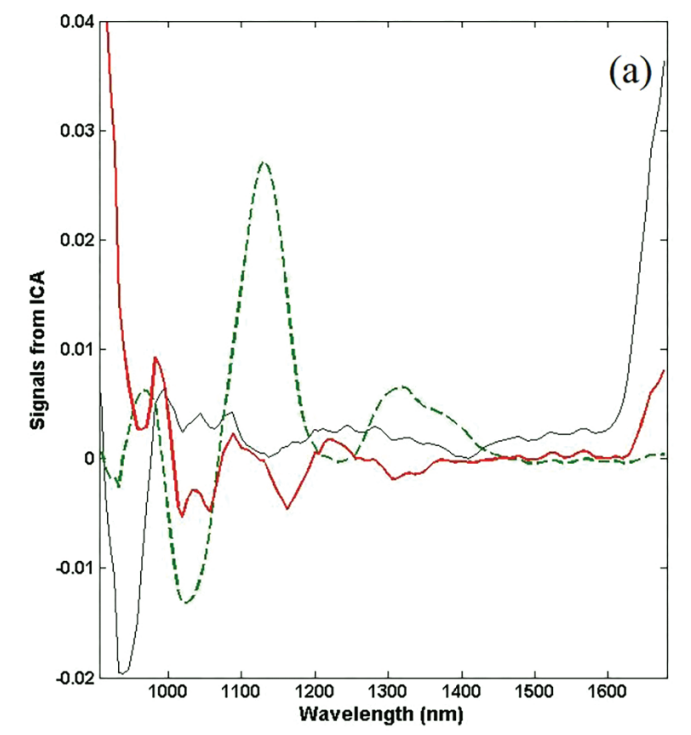

parentheses correspond to the number of samples employed in the pseudo-univariate calibration curve): in orange juice determination of carbendazim (4 samples), ${ }^{11}$ and in the evaluation of antioxidant activity in degreased chia seeds extracts (4 samples), ${ }^{26}$ in both cases based on NIR spectroscopy. Pseudo-univariate calibration models were also useful in the ascorbic acid determination from powder juices by using UV-Vis absorption spectral data (3 samples), and in the determination of tetracycline in serum samples using fluorescence emission spectroscopy (4 samples), ${ }^{16}$ and the quantification of nicotine in urine samples (6 samples). ${ }^{14}$

On the other hand, anthocyanins were quantified based on NIR spectra and PLS from intact açai (Euterpe oleracea Mart.) and palmitero-juçara (Euterpe edulis Mart.), and in both cases, a total of 139 samples were employed in the calibration development. ${ }^{24}$ NIR hyperspectral imaging coupled to PLS were used to determine anthocyanins in wine, and in this case, a total of 120 samples were considered. ${ }^{27}$

From the presented, possibilities concerning of employing a reduced number of samples, no interferents inclusion in the calibration step, seems to make pseudounivariate calibrations a trend in analytical chemistry, and here the results showed that NIR spectra coupled to ICA allowed the direct quantification of TA and AA in whole grape juices. Furthermore, for TA determination two different buffer systems are needed. Although the reference method for TA quantification consists only on add just $1 \mathrm{~mL}$ of two buffers in low concentration and register the absorbance using a UV spectrophotometer, it is important to highlight the time demanded and possibilities of errors in the step of the buffer preparation. Moreover, more steps in the method imply

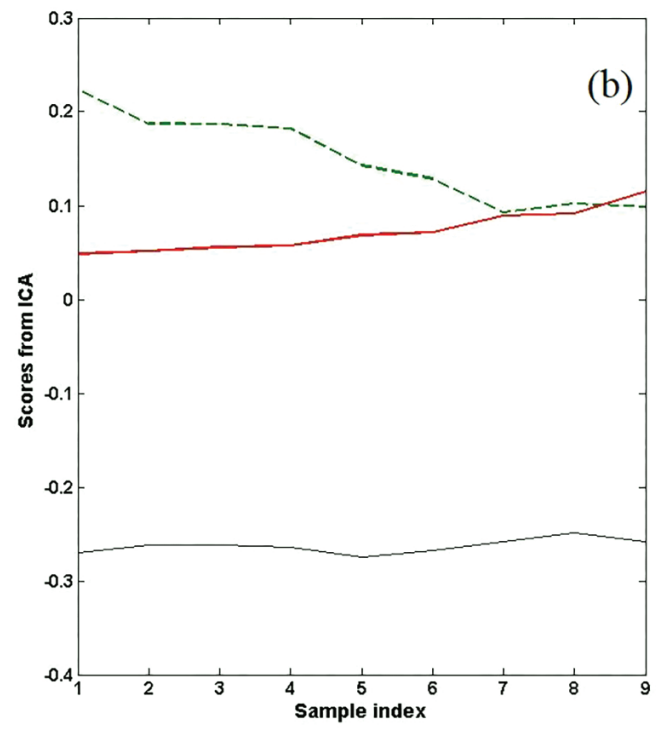

Figure 4. ICA results: (a) signals and (b) scores. (-) IC1: interferent, (---) IC2: antioxidants, (-) IC3: anthocyanins. 

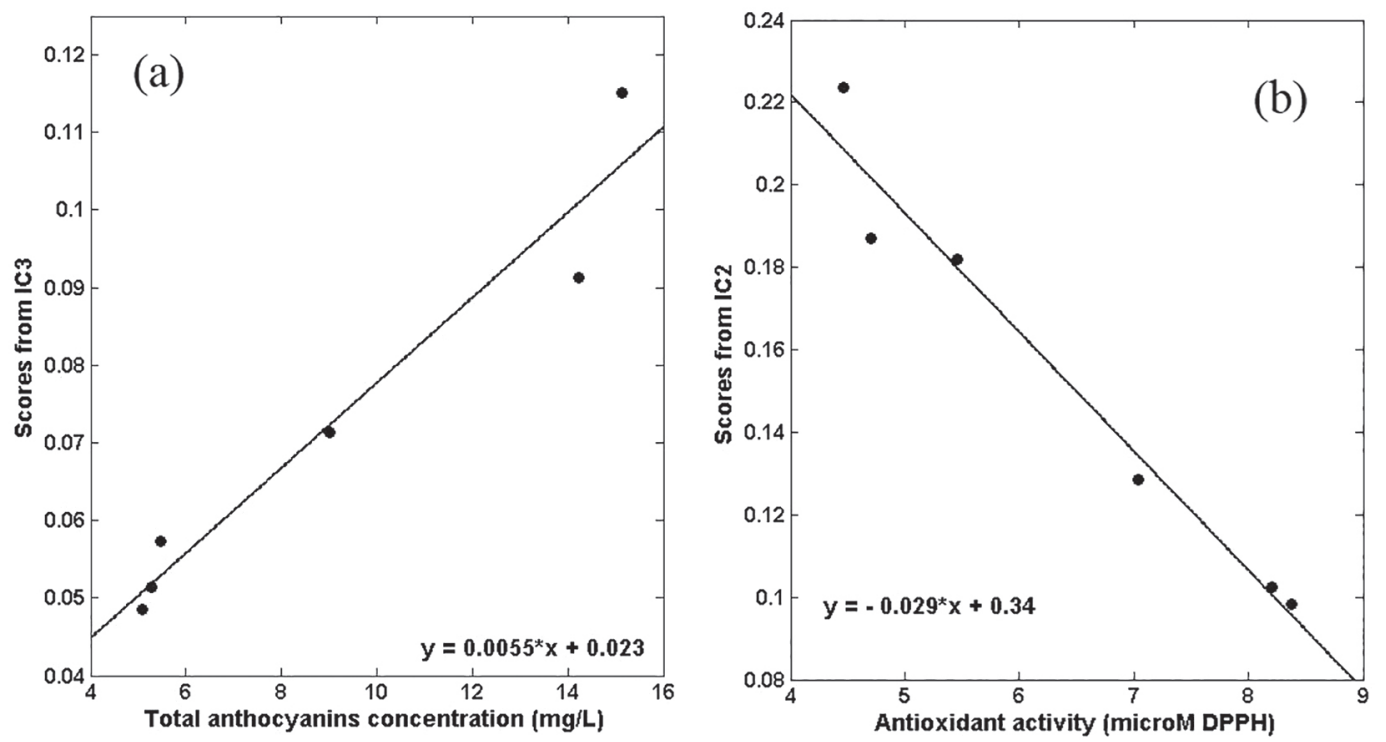

Figure 5. Pseudo-univariate calibration curve for determination of total anthocyanins (a) and antioxidant activity (b).

Table 2. Results for the prediction of TA and AA by pseudo-univariate calibration model

\begin{tabular}{lccccccc}
\hline $\begin{array}{l}\text { TA by reference } \\
\text { methodology / } \\
\left(\mathrm{mg} \mathrm{L}^{-1}\right)\end{array}$ & $\begin{array}{c}\text { Scores from } \\
\text { IC3 related to } \\
\text { anthocyanins }\end{array}$ & $\begin{array}{c}\text { Predicted TA / } \\
\left(\mathrm{mg} \mathrm{L}^{-1}\right)\end{array}$ & Absolute error & $\begin{array}{c}\text { AA by reference } \\
\text { methodology / } \\
(\mu \mathrm{M} \mathrm{DPPH})\end{array}$ & $\begin{array}{c}\text { Scores from } \\
\text { IC2 related to } \\
\text { antioxidants }\end{array}$ & $\begin{array}{c}\text { Predicted AA / } \\
(\mu \mathrm{M} \text { DPPH })\end{array}$ & $\begin{array}{c}\text { Absolute error } \\
5.37\end{array}$ \\
0.0554 & 5.89 & -0.52 & 5.29 & 0.1900 & 5.17 & 0.12 \\
8.82 & 0.0685 & 8.27 & 0.55 & 5.54 & 0.1424 & 6.81 & -1.27 \\
11.02 & 0.0890 & 12.00 & -0.98 & 7.96 & 0.0927 & 8.53 & -0.57 \\
\hline
\end{tabular}

TA: total anthocyanins; AA: antioxidant activity; DPPH: 2,2-diphenyl-1-picrylhydrazyl.

more error propagation. So, when the pseudo-univariate calibration model is ready and validated, these steps are no more necessary, and this is an advantage in using the chemometric strategy that overcomes the univariate one.

On the other hand, for AA it is necessary to build a calibration curve by using DPPH reagent, and measurement should be done in a dark environment. So, the reference methods use reagents/solvents, are time-consuming, lead to waste that should be appropriately disposed of. With this proposal, once the pseudo univariate calibration model is developed, it is only necessary a NIR spectrum of the whole grape juice.

\section{Conclusions}

NIR spectroscopy combined with the ICA chemometric tool allows the development of pseudo-univariate calibration models by plotting ICA scores against anthocyanins and antioxidants content obtained by reference methodologies. The calibration results suggest that by combining NIR spectra and ICA the direct quantification of TA and AA in matrices with unknown interferents, like grape juice, is achieved. However, overcome interferences using first- order data are possible and work properly when there is enough selectivity for the analyte profile in the sample data. Furthermore, the proposal employs a smaller number of samples in the calibration step, besides being free of sample preparation.

\section{Acknowledgments}

The authors thank Coordenação de Aperfeiçoamento de Pessoal de Nível Superior (CAPES), and Fundação Araucária (process 033/2019).

\section{Author Contributions}

Karla K. Beltrame and Thays R. Gonçalves were responsible for data curation, formal analysis, investigation, software, validation, visualization, writing the original draft; Paulo H. Março, Sandra T. M. Gomes and Makoto Matsushita for conceptualization, funding acquisition, investigation, project administration; Patrícia Valderrama for conceptualization, investigation, project administration, resources, software, validation, visualization, writingreview and editing. 


\section{References}

1. Shahidi, F.; Ambigaipalan, P.; J. Funct. Foods 2015, 18, 820.

2. Albishi, T.; John, J. A.; Al-Khalifa, A. S.; Shahidi, F.; J. Funct. Foods 2013, 5, 930.

3. Sun, Y.; Hu, X.; Li, W.; Int. J. Biol. Macromol. 2017, 97, 323.

4. Toaldo, I. M.; Cruz, F. A.; Silva, E. L.; Bordignon-Luiz, M. T; Nutr. Res. 2016, 36, 808.

5. Stafussa, A. P.; Maciel, G. M.; Anthero, A. G. S.; Silva, M. V.; Zielinski, A. A. F.; Haminiuk, C. W. I.; J. Food Eng. 2016, 169 , 53.

6. Sang, J.; Sang, J.; Ma, Q.; Hou, X.-f.; Li, C.-q.; Food Chem. 2017, 218, 386.

7. Peron, D.V.; Fraga, S.; Antelo, F.; Food Chem. 2017, 232, 836.

8. Aji Muhammad, D. R.; Praseptiangga, D.; Walle, D. V.; Dewettinck, K.; Food Chem. 2017, 231, 356.

9. Romera-Fernández, M.; Berrueta, L. A.; Garmón-Lobato, S.; Gallo, B.; Vicente, F.; Moreda, J. M.; Talanta 2012, 88, 303.

10. Mariani, N. C. T.; Teixeira, G. H. A.; Lima, K. M. G.; Morgenstern, T. B.; Nardini, V.; Júnior, L. C. C.; Food Chem. 2015, 174, 643.

11. Ribeiro, G. M.; Madivadua, D. A.; Curti, S. M. M.; Pantean, L. P.; Março, P. H.; Valderrama, P.; Microchem. J. 2017, 134, 114.

12. Fu, H.; Yin, Q.; Xu, L.; Wang, W.; Chen, F.; Yang, T.; Spectrochim. Acta, Part A 2017, 182, 17.

13. Hindle, P. H. In Handbook of Near-Infrared Analysis; Burns, D. A.; Ciurczak, E. W., eds.; CRC Press: Boca Raton, USA, 2008, ch. 1 .
14. Mamián-López, M. B.; Poppi, R. J.; Anal. Chim. Acta 2013, $760,53$.

15. Mohseni, N.; Bahram, M.; Olivieri, A. C.; Spectrochim. Acta, Part A 2014, 122, 721.

16. Goicoechea, H. C.; Olivieri, A. C.; Tauler, R.; Analyst 2010 , 135, 636.

17. Lee, J.; Durst, R. W.; Wrolstad, R. E.; J. AOAC Int. 2005, 88, 1269.

18. Farhadi, K.; Esmaeilzadeh, F.; Hatami, M.; Forough, M.; Molaie, R.; Food Chem. 2016, 199, 847.

19. Kim, Y. K.; Guo, Q.; Packer, L.; Toxicology 2002, 172, 149.

20. MATLAB, version R2007b; The MathWorks, Inc.: Natick, MA, USA, 2007.

21. Bouveresse, D. J.-R.; Rutledge, D. N.; TrAC, Trends Anal. Chem. 2013, 50, 22.

22. Bouveresse, D. J.-R.; Rutledge, D. N.; TrAC, Trends Anal. Chem. 2015, 67, 220.

23. Savitzky, A.; Golay, M. J. E.; Anal. Chem. 1964, 36, 1627.

24. Inácio, M. R. C.; Lima, K. M. G.; Lopes, V. G.; Pessoa, J. D. C.; Teixeira, G. H. A.; Food Chem. 2013, 136, 1160.

25. Março, P. H.; Poppi, R. J.; Scarminio, I. S.; Tauler, R.; Food Chem. 2011, 125, 1020.

26. Lazkowski, M. S.; Gonçalves, T. R.; Gomes, S. T. M.; Março, P. H.; Valderrama, P.; Matsushita, M.; LWT-Food Sci. Technol. 2018, 95, 303.

27. Chen, S.; Zhang, F.; Ning, J.; Liu, X.; Zhang, Z.; Yang, S.; Food Chem. 2015, 172, 788. 